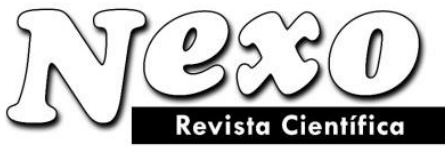

ISSN-E 1995-9516

Universidad Nacional de Ingeniería http://revistas.uni.edu.ni/index.php/Nexo http://dx.doi.org/10.5377/nexo.v29i2.4572

Vol. 29, No. 02, pp. 44-58/Diciembre 2016

(c) (i) $\circledast$

\title{
INSULINA ESTIMULA LA PROLIFERACIÓN CELULAR DEPENDIENTE DE AUXINAS EN LOS CULTIVOS DE CÉLULAS EN SUSPENSIÓN DE TABACO NT-1
}

\section{INSULIN STIMULATES AUXIN-DEPENDENT CELL PROLIFERATION IN NT-1 TOBACCO SUSPENSION CELL CULTURES}

\author{
G. Fierros-Romero ${ }^{1 *}$, M. E. Mellado-Rojas ${ }^{1}$, E. M. Beltrán-Peña ${ }^{1}$ \\ Laboratorio de Transducción de señales; Instituto de Investigaciones Químico Biológicas; Universidad \\ Michoacana de San Nicolás de Hidalgo; Michoacán, México. \\ *griselfierrosromero@gmail.com
}

(recibido/received: 16-Agosto-2016; aceptado/accepted: 01-Octubre-2016)

\begin{abstract}
RESUMEN
La insulina regula el crecimiento en plantas como maíz y frijol, a través de la proliferación celular. En este estudio observamos que en cultivos de tabaco NT-1, la hormona regula la proliferación celular de manera dependiente de la presencia de auxinas. Los cultivos donde se limitó la concentración de auxinas, presentaron una baja actividad mitótica, la cual fue re establecida por la adición de $1.23 \mathrm{nM}$ de insulina a través de la expresión de los genes ciclina $B, C D K B, E 2 F$, subunidad $\beta$ - de la proteína $G A R C A$ y glutation$S$-transferasa $(G S T)$. Los cultivos también presentaron un incremento en peso seco y células pequeñas que corresponden a un fenotipo con alta división celular. Por otra parte, se ha reportado que la ausencia de auxinas en este tipo de cultivos de células de tabaco en suspensión conduce a una sincronización parcial, y en este sistema la insulina también promovió la proliferación, incrementando la expresión del gen E2F.
\end{abstract}

Palabras clave: Insulina, auxinas, proliferación, cultivos NT-1.

\begin{abstract}
Insulin regulates growth in plants such as maize and beans, through cell proliferation. In this study we observed that in NT-1 tobacco cell cultures, the hormone regulates cell proliferation dependent on the presence of auxins. The cultures where the auxin concentration was limited showed a low mitotic activity, which was reestablished by the addition of $1.23 \mathrm{nM}$ of insulin through the expression of the cyclin $B, C D K B$, $E 2 F, G$ protein subunit $\beta$ ARC A and glutation -S-transferase (GST) genes. The cultures also presented an increase in dry weight and small cells corresponding to a phenotype with high cell division. On the other hand, it has been reported that the absence of auxins in this type of culture of suspended tobacco cells leads to a partial synchronization, and in this system insulin also promoted proliferation, increasing the expression of the E2F gene.
\end{abstract}

Keywords: Insulin, auxin, proliferation, NT-1 cells. 


\section{G. Fierros-Romero et al.}

\section{INTRODUCCIÓN}

En metazoarios, el crecimiento y la proliferación celular son procesos regulados por insulina y factores de crecimiento parecidos a insulina (IGF) a través de dos cascadas de transducción de señales: la vía del fosfatidil inositol-3-cinasa (PI3K)-TOR y la de proteínas cinasas activadas por mitógenos (MAPK). La activación de estas rutas modula en forma coordinada el tráfico vesicular, la síntesis de proteínas, la activación de enzimas y la expresión genética, lo que resulta en una regulación del metabolismo de la glucosa, lípidos y proteínas (Saltiel y Kahn, 2001).

En cuanto a las plantas, se ha reportado que la insulina podría estar involucrada en su crecimiento, debido a que sustancias parecidas a la insulina inicialmente denominadas "glucocininas" se encontraron en cebollas, hojas de lechuga, raíces de cebada, remolacha, germinados de papa y arroz (Best, 1923; Collip, 1923). Mientras que en semillas de pepino, sandía y girasol en presencia de insulina se observó una emergencia acelerada de la radícula, sugiriendo con ello que dicha hormona presenta un papel regulador tanto en plantas como en animales (Goodman y Davis, 1993). En otros estudios se reportó que la insulina aceleraba la germinación del maíz promoviendo el reclutamiento a polisomas del transcrito de la proteína ribosomal S6 y el incremento en el nivel de fosforilación de dicha proteína (Sánchez de Jiménez et al., 1999). En extractos de maíz germinado por 46 horas se purificó y caracterizo una proteína a la cual se le denomino factor de crecimiento parecido a insulina de maíz (ZmIGF) con un peso aproximado de $6 \mathrm{kDa}$ y con actividad y estructura similar a la insulina de bovino (Garcia Flores et al., 2001; Rodriguez-Lopez et al., 2011). Se observó que dicha proteína estimulo el crecimiento y la división celular en maíz, promoviendo la síntesis de DNA y proteínas ribosomales (Rodriguez-Lopez et al., 2011).

En un estudio reciente se analizó la expresión de genes durante la germinación en ejes embrionarios de maíz de entre las 0 y $24 \mathrm{~h}$ a través de un ensayo con micro arreglos. Agrupándose dichos genes en diferentes categorías relacionadas al metabolismo, transporte, transcripción, traducción, entre otros. Los genes que codifican para proteínas ribosomales varían muy poco durante este periodo de germinación y necesitan de la formación del ribosoma, lo que sugiere que estos genes son regulados a nivel traduccional. Analizando la expresión genética del RNAm polisomal de ejes embrionarios germinados estimulados y no estimulados con insulina, se observó mayor reclutamiento a polisomas de transcritos de proteínas ribosomales en los tejidos estimulados con insulina, indicando un mecanismo de control traduccional para permitir la rápida síntesis de proteínas ribosomales (Jimenez-Lopez et al., 2011).

Es bien conocido que el crecimiento y el desarrollo en plantas está determinado por procesos como la división, la elongación y la diferenciación, los cuales son regulados por los fitorreguladores, principalmente las auxinas (Perrot-Rechenmann, 2010). Durante la división celular, la célula transcurre por dos etapas: la mitosis y la interfase (G1, S y G2), donde el ciclo es regulado en las interfases G1/S y G2/M por los complejos de ciclinas y cinasas dependientes de ciclinas, que a su vez son modulados por las auxinas, citocininas, brasinoesteroides entre otros efectores. Durante la transición G1/S, principalmente el complejo ciclina D/CDKA fosforila a la proteína RBR, liberando entonces al factor de transcripción E2F que se encuentra formando parte del complejo inactivo RBR/E2F, dicho factor promueve la transcripción de los genes necesarios para la síntesis de DNA. Mientras que en la transición G2/M interviene el complejo ciclina B/CDKA/CDKB (Gutierrez, 2009). En las plantas y específicamente en la raíz, la elongación celular ocurre fuera de la zona de división, al incrementar la presión de turgencia mediante el alargamiento de la vacuola por el ingreso de agua y la síntesis de novo de los componentes de la pared celular, tales procesos son también dependientes de la acción de las auxinas (Mockaitis y Estelle, 2008).

Las auxinas poseen una función central en el crecimiento y desarrollo de la planta, siendo considerada como hormonas morfogénicas dosis-dependiente. La percepción de las auxinas en las células ha estado sujeta a numerosas investigaciones, donde se ha mostrado la existencia de dos tipos de receptores; uno membranal como la proteína de unión a auxina (ABP1) y otro nuclear como la proteína resistente a inhibidores de

Nexo Revista Científica / Vol. 29, No. 02, pp. 44-58 / Diciembre 2016 


\section{G. Fierros-Romero et al.}

transporte (TIR1). ABP1 media la elongación de la célula al activar a una bomba ATPasa y canales de $\mathrm{K}^{+}$, estos promueven la entrada de agua lo que da como resultado la expansión celular (Simon and Petrasek, 2011). Mientras que. la unión de las auxinas a TIR1 conduce a una rápida activación de la transcripción de genes de respuesta temprana a auxina dependiente de la degradación de represores AUX/IAA por el sistema de ubiquitinación (Mockaitis y Estelle, 2008).

Los cultivos de células en suspensión de tabaco, son sistemas relativamente homogéneos y por lo tanto adecuados para estudiar los mecanismos moleculares involucrados en la regulación del crecimiento vegetal, tales como la proliferación y elongación celular. En cultivos en suspensión de tabaco BY-2 y NT-1 se requiere la aplicación de auxinas exógenas como el 2,4-diclorofenoxiacético (2,4-D) para mantener la división celular (Raghavan, 2004). Interesantemente, cuando las células vegetales de tabaco carecen de auxina se presenta un proceso de elongación muy marcado (Winicur et al., 1998). Se ha reportado que las auxinas a concentraciones bajas en el medio (menos de $1 \mu \mathrm{M}$ ) promueven la expansión celular, mientras que concentraciones arriba de $1 \mu \mathrm{M}$ estimulan la división celular (Chen et al., 2001; Nagata et al., 2004), siendo entonces los cultivos de tabaco NT-1 un excelente modelo para entender los procesos de proliferación y elongación regulados por auxinas. Debido a que estas últimas, promueven la proliferación en cultivos de tabaco en suspensión y se ha mostrado que la insulina estimula la proliferación en maíz, en el presente estudio se decidió evaluar la posible interacción de ambas moléculas en los cultivos suplementados con bajas concentraciones de auxinas que limitan su actividad mitótica.

\section{MATERIALES Y MÉTODOS}

\subsection{Mantenimiento del cultivo celular NT-1}

Los cultivos de células de tabaco en suspensión NT-1 se mantuvieron en oscuridad, con agitación a 100 rpm y $25{ }^{\circ} \mathrm{C}$, por subcultivos semanales inoculando $3.5 \mathrm{~mL}$ de células de un cultivo previo en fase estacionaria (día siete) a $50 \mathrm{~mL}$ de medio Murashige and Skoog $1 \mathrm{X}\left(4.3 \mathrm{~g} / \mathrm{l}\right.$ de $\mathrm{MS}, 30 \mathrm{~g} / \mathrm{l}$ de sacarosa, $0.2 \mathrm{~g} / \mathrm{l}$ de $\mathrm{KH}_{2} \mathrm{PO}_{4}$, $0.9 \mathrm{nM}$ de 2,4-D, $100 \mathrm{mg} / \mathrm{l}$ de mio-inositol y $1 \mathrm{mg} / \mathrm{l}$ de tiamina). En condiciones asépticas, se filtró el total del cultivo de células en suspensión en fase estacionaria de 7 días y se lavó con 11 de medio MS $1 \mathrm{X}$ ( $\sin$ vitaminas ni hormona). Las células se resuspendieron en $100 \mathrm{~mL}$ de medio MS 1X y de dicho cultivo se tomaron alícuotas para inocular los diferentes tratamientos. Para realizar las cinéticas de crecimiento, se inocularon $100 \mathrm{~mL}$ de medio MS $1 \mathrm{X}$ (suplementado con $0.123,1.23,12.3 \mathrm{nM}$ de insulina y $0,0.225$ y 0.45 $\mu \mathrm{M}$ de 2,4-D) con $7 \mathrm{~mL}$ del inóculo antes mencionado, se incubaron y tomaron alícuotas diarias de $3 \mathrm{~mL}$.

Para obtener las cinéticas de crecimiento de los cultivos semi sincronizados se inocularon con $7 \mathrm{~mL}$ de células, $100 \mathrm{~mL}$ medio MS sin 2,4-D y se incubaron durante 4 días. Transcurrido este tiempo, de dicho cultivo se transfirieron $7 \mathrm{~mL}$ a $100 \mathrm{~mL}$ de medio MS suplementado con $0.9 \mu \mathrm{M}$ de 2,4-D (concentración habitual para el mantenimiento de los cultivos NT-1) y $1.23 \mathrm{nM}$ de insulina.

\subsection{Evaluación del crecimiento}

El Índice Mitótico se determinó fijando las células con una solución de paraformaldehido al 4\% y glutaraldehido al $50 \%$ durante $12 \mathrm{~h} \mathrm{a} 4{ }^{\circ} \mathrm{C}$. Una vez que se retiró el sobrenadante, se agregó $0.5 \mathrm{~mL}$ de DAPI (10mg de 4',6-diamino-2 fenilindol /mL PBS). El índice mitótico se determinó por microscopía de epifluorescencia usando la formula siguiente: \% IM = Total de células en mitosis / 500 células contadas X 100. El tamaño celular se determinó capturando imágenes de las células NT-1 teñidas con DAPI con una cámara Nikon Coolpixs10 acoplada al microscopio de epifluorescencia Nikon. Se midió la longitud y el ancho de las células con ayuda del programa ImageJ (Mravec et al., 2008).

\subsection{RT-PCR semi cuantitativo}

Nexo Revista Científica / Vol. 29, No. 02, pp. 44-58 / Diciembre 2016 


\section{G. Fierros-Romero et al.}

Se trituraron 500 mg de células NT-1 con nitrógeno líquido y se extrajo el RNA por el método del Trizol (Invitrogen). Se utilizó el Kit SuperScript III ${ }^{\text {TM}}$ One-Step RT-PCR Systemwith Platinum ${ }^{\circledR}$ taq DNA polymerase, en base a las especificaciones establecidas por la casa comercial Invitrogen ${ }^{\mathrm{TM}}$. Las reacciones fueron efectuadas con RNA $(0.64 \mu \mathrm{g} / \mu \mathrm{l})$.

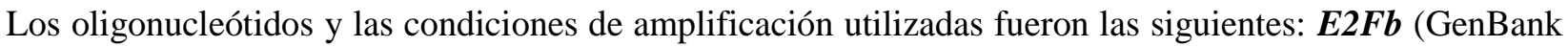
AB025347): sentido 5'CAAATTACAAACAGGGAGTTGGTGC 3' y antisentido $5^{\prime}$ CTCCTTTGTGGATAATCAACAGCCT $3^{\prime}$. Las condiciones del RT-PCR $30 \mathrm{~min}$ a $50^{\circ} \mathrm{C}, 94^{\circ} \mathrm{C} 5 \mathrm{~min}$ y 35 ciclos de $15 \mathrm{seg}$ a $94{ }^{\circ} \mathrm{C}, 1 \mathrm{~min}$ a $55{ }^{\circ} \mathrm{C}$ y $45 \mathrm{seg}$ a $68^{\circ} \mathrm{C}$. CDKB (GenBank. X57840): sentido 5'GGGTCTTGGTCGTGCTTTTACTGTTT 3' y antisentido 5'CCAAGACGATGACA ACAGATACAGC $3^{\prime}, 30 \mathrm{~min}$ a $50^{\circ} \mathrm{C}, 5 \mathrm{~min}$ a $94^{\circ} \mathrm{C}$ y 40 ciclos de $15 \mathrm{seg}$ a $94^{\circ} \mathrm{C}, 1 \mathrm{~min}$ a $50^{\circ} \mathrm{C}$ y $45 \mathrm{seg}$ a $68^{\circ} \mathrm{C}$. Ciclina B (GenBank. D89635.1): sentido 5'ATAACGAGGGGCTTTTGTGC3' y antisentido $5^{\prime} \mathrm{CTTTTCTCCATTCCCAACACCT3} 3^{\prime}, 30 \mathrm{~min}$ a $50^{\circ} \mathrm{C}, 5 \mathrm{~min}$ a $94^{\circ} \mathrm{C}$ y 25 ciclos de $30 \mathrm{seg}$ a $95^{\circ} \mathrm{C}, 30 \mathrm{seg}$ a $\quad 60^{\circ} \mathrm{C}$ y 30 seg a $72^{\circ} \mathrm{C}$. Glutatión-S-transferasa (GenBank. D10524.1): sentido 5’TGCAACCATGAGAGTTGCTGCTTG 3' y antisentido 5'ATTGCGCTTCGC TTTCCTTCACAG $3^{\prime}, 30 \mathrm{~min}$ a $50^{\circ} \mathrm{C}, 5 \mathrm{~min}$ a $94^{\circ} \mathrm{C}$ y 25 ciclos de $30 \mathrm{seg} .95^{\circ} \mathrm{C}, 30 \mathrm{seg}$ a $65^{\circ} \mathrm{C}$ y $30 \mathrm{seg}$ a $72^{\circ} \mathrm{C}$. Subunidad B$\begin{array}{llllllll}\boldsymbol{G} & \boldsymbol{A} \boldsymbol{R} \boldsymbol{C} & \boldsymbol{A} & \text { (GenBank. D17526.1): sentido 5'TGAGCTCCGTTTGTGGGATCTTCA } & 3^{\prime} & \text { y }\end{array}$ antisentido5'ACTCCAGCTCAAACTGGTGCAGTA $3^{\prime}, 30 \mathrm{~min}$ a $50^{\circ} \mathrm{C}, 5 \mathrm{~min}$ a $94^{\circ} \mathrm{C}$ y 25 ciclos de 30 seg a $95^{\circ} \mathrm{C}, \quad 30 \mathrm{seg}$ a $65^{\circ} \mathrm{C}$ y $30 \mathrm{seg}$ a $72^{\circ} \mathrm{C}$. Actima (GenBank. X63603): sentido 5'CCTCTTAACCCGAAGGCTAA 3' yantisentido5'GAAGGTTGGAAAAGGACTTC 3', 30 min a 50 ${ }^{\circ} \mathrm{C}$, $5 \min$ a $94^{\circ} \mathrm{C}$ y 25 ciclos de $15 \mathrm{seg}$ a $94^{\circ} \mathrm{C}, 1 \mathrm{~min}$ a $55^{\circ} \mathrm{C}$ y $45 \mathrm{seg}$ a $68^{\circ} \mathrm{C}$.

\subsection{Análisis de datos}

Se utilizó el programa STATISTICA ver 8.0 para analizar los resultados de los experimentos presentados a través de una prueba de Tukey. Las diferentes letras indican los tratamientos que difieren estadísticamente $(\mathrm{P} \leq 0.05)$.

\section{RESULTADOS}

\subsection{Proliferación celular de los cultivos NT-1 por efecto de la insulina}

Para evaluar directamente el efecto de la insulina sobre la proliferación celular, se calculó el porcentaje de Índice Mitótico (\% IM) al día seis correspondiente a la fase log de la cinética de crecimiento (Figura 1A), donde observamos que $1.23 \mathrm{nM}$ de insulina aumentó en un $36 \%$ el IM respecto al control. Además se evaluó el tamaño de las células, debido a que células pequeñas en este tipo de cultivos se relaciona directamente con la proliferación celular (Matsuoka et al., 2004). Aquí, encontramos que la mayoría de las células NT-1 tratadas con $1.23 \mathrm{nM}$ de insulina son pequeñas comparadas con el control (Figura 1B). Lo anterior se observa también en las fotografías representativas de células teñidas con DAPI, donde las células más pequeñas corresponden al tratamiento con $1.23 \mathrm{nM}$ de insulina (Figura 1C). Los parámetros antes analizados corresponden a la fase de crecimiento exponencial de las cinéticas de crecimiento de los cultivos NT-1, evaluadas por peso seco y Volumen de Paquete Celular (VPC). Dichas cinéticas presentan una fase lag de adaptación hasta el tercer día, una fase log del día tres al siete y finalmente la estacionaria a partir del día siete (Figura suplementaria 1). En las diferentes fases de las cinéticas (lag, log y estacionaria), las células presentan variaciones morfológicas, largas en la fase lag y estacionaria y pequeñas en la fase log (Figura suplementaria 1C). En relación al efecto de diferentes concentraciones de insulina, observamos que la proliferación de los cultivos NT-1 fue estimulada por la concentración de $1.23 \mathrm{nM}$ (Figura 2A), efecto que también fue determinado a través dela velocidad específica de crecimiento (Figura suplementaria 2B). Estos resultados sugieren que las células NT-1 bajo el efecto de $1.23 \mathrm{nM}$ de insulina proliferan con mayor velocidad. 


\section{G. Fierros-Romero et al.}

A

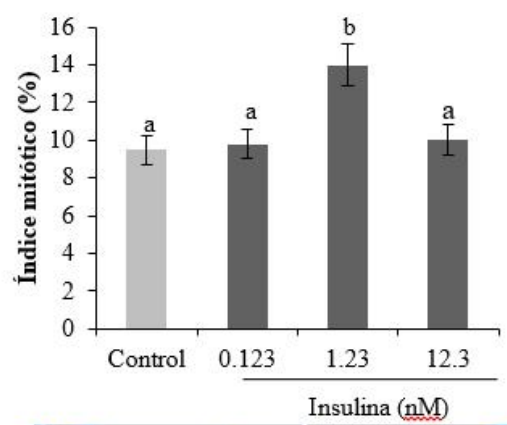

B

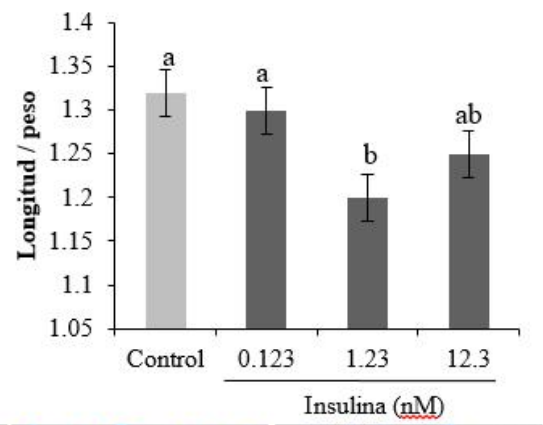

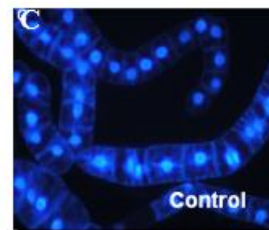
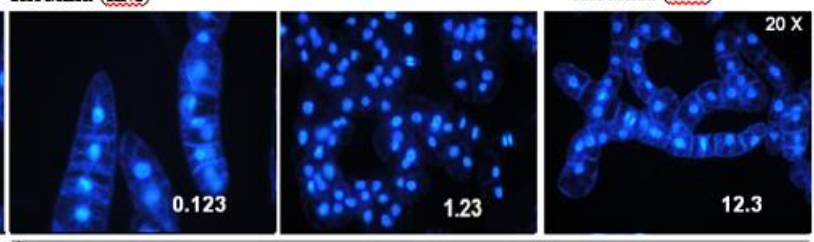

Insulina (nM)

Figura 1. Efecto de la insulina sobre la proliferación y el tamaño celular. A) Porcentaje de Índice Mitótico (IM) determinado en el día seis correspondiente a la fase log de las cinéticas de crecimiento de los cultivos NT-1. B) Tamaño celular. Las células teñidas con DAPI se midieron para calcular la relación largo/ancho. C) Fotografías de células NT1 en fase $\log$ teñidas con DAPI tomadas en un microscopio de epifluorescencia con un objetivo 20X. Tukey. $\mathrm{P} \leq 0.05$; $\mathrm{n}=4$, STATISTICA ver. 8.0

\subsection{El estímulo de la insulina sobre la proliferación de los cultivos NT-1requiere la presencia de 2,4-D}

Debido a que se observó que $1.23 \mathrm{nM}$ de insulina fue capaz de estimular la proliferación en los cultivos suplementados con $0.9 \mathrm{nM}$ de auxinas, se decidió evaluar si la hormona podría inducir la proliferación celular en ausencia de auxinas. Se ha descrito en cultivos de tabaco en suspensión que concentraciones alrededor de $1 \mu \mathrm{M}$ de auxinas promueven la proliferación, mientras que concentraciones más bajas inducen la expansión celular (Nagata et al., 2004).

Se puede apreciar que en las cinéticas de crecimiento de los cultivos suplementados con $0.123,1.23$ y 12.3 $\mathrm{nM}$ de insulina sin auxinas, ninguna de las concentraciones de insulina promovió un aumento en peso seco (Figura 2A). Además, los cultivos sin auxinas presentaron una actividad mitótica (IM) alrededor de 2.5\%, lo que representa una disminución aproximada de un $80 \%$ (Figura 2B) respecto a los cultivos crecidos en presencia de auxinas, que fue alrededor del 10\% (Figura 1A). Además, se observa en estos cultivos células alargadas en todos los tratamientos, como consecuencia de la ausencia de auxinas (Figuras 2C y 2D). Estos datos indican que la insulina requiere la presencia de las auxinas para estimular la proliferación de los cultivos NT-1. 


\section{G. Fierros-Romero et al.}

A
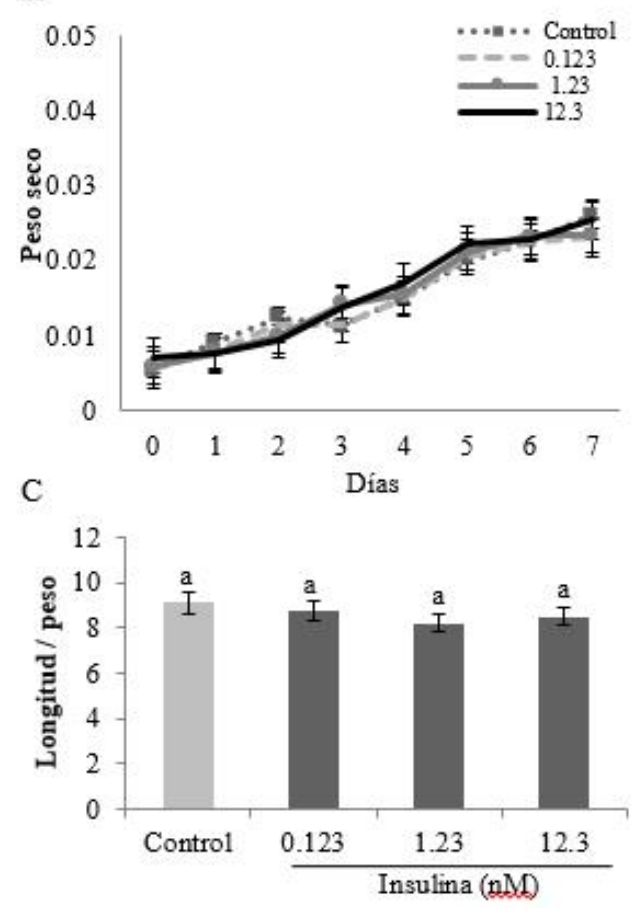

B

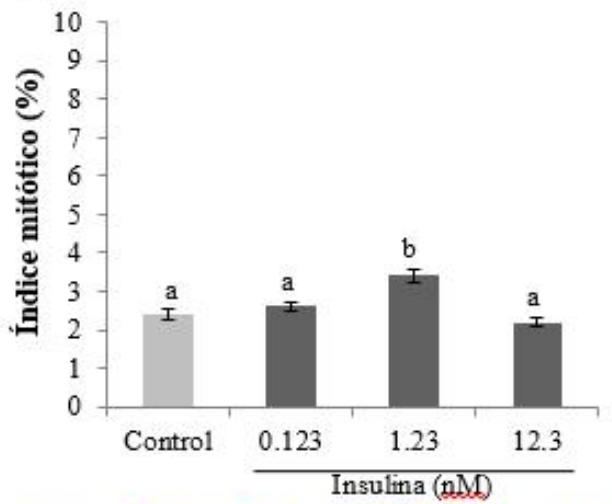

D

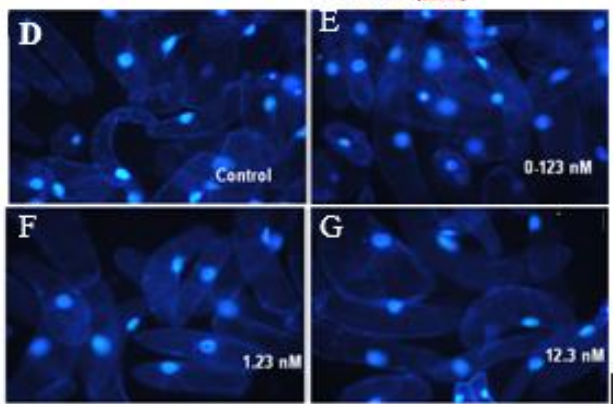

Figura 2. Efecto de la insulina sobre el crecimiento de los cultivos NT-1 en medio sin auxina. A) Cinéticas de crecimiento de los cultivos suplementados con $0.123,1.23,12.3$ y $61.5 \mathrm{nM}$ de insulina en medio que carece de 2,4-D valuadas por peso seco. B) Porcentaje de Índice Mitótico (IM) determinado en el día seis de fase log. C) Tamaño celular. Las células teñidas con DAPI se midieron para calcular la relación largo/ancho con el programa ImageJ. Fotografías de células NT-1 en fase log teñidas con DAPI: D) control, E) $0.123 \mathrm{nM}$ de insulina, F) $1.23 \mathrm{nM}$ y G) 12.3 nM. Tukey. $\mathrm{P} \leq 0.05 ; \mathrm{n}=4$ STATISTICA ver. 8.0

\subsection{La insulina estimula la proliferación celular en cultivos con baja concentración de 2,4-D}

Una vez que se determinó que la insulina para estimular la proliferación de los cultivos NT-1 requiere de las auxinas, se decidió investigar el efecto de dicha hormona en un sistema con actividad mitótica restringida por efecto de una baja concentración de auxinas en el medio. Como se mencionó anteriormente, $0.9 \mu \mathrm{M}$ es la concentración óptima de 2,4-D para el mantenimiento de los cultivos NT-1, así que se ensayaron concentraciones de 0.45 y $0.225 \mu \mathrm{M}$ de 2,4-D. Debido a que las células en estas condiciones incrementan su volumen como consecuencia de la elongación (Nagata et al., 2004), se decidió evaluar el crecimiento mediante el parámetro del VPC, además del peso seco.

En la Figura 3A se muestra un incremento de VPC en los cultivos adicionados con $0.225 \mu \mathrm{M}$ de auxina a diferencia del peso seco que no presentó diferencias significativas. Además, la literatura indica que las auxinas regulan la proliferación celular en cultivos BY-2 de manera dosis dependiente (Nagata et al., 2004). Por lo anterior, se determinó el IM en los cultivos suplementados con las diferentes concentraciones de auxinas, para seleccionar la concentración que limitara la proliferación y determinar en ellos, si dicho parámetro se restablecía con la insulina. Podemos observar en la Figura 3B que el IM disminuyó alrededor de un $40 \%$ en los cultivos con 0.45 y $0.225 \mu \mathrm{M}$ de auxinas respecto al control. En relación al tamaño, se muestra que las células con $0.225 \mu \mathrm{M}$ de 2,4-D lo incrementaron significativamente en más de 300\% (Figuras 3C y 3D), lo que además coincide con el incremento del VPC en los cultivos suplementados con $0.225 \mu \mathrm{M}$ de 2,4-D (Figura 3A).

Se ha reportado que el incremento en la elongación celular en este tipo de cultivos es ocasionada por una escasa actividad mitótica (Chen et al., 2001; Nagata, 1992). Por los resultados antes obtenidos, se seleccionó 


\section{G. Fierros-Romero et al.}

$0.225 \mu \mathrm{M}$ de 2,4-D como la concentración limitante para la proliferación celular en cultivos NT-1 y se adicionaron con $1.23 \mathrm{nM}$ de insulina. Se observó que la hormona estimuló el peso seco a partir del cuarto día de la fase log (Figura 4A), mientras que la cinética evaluada por VPC (Figura 4B) muestra la tendencia a disminuir dicho parámetro en los cultivos tratados con insulina, sin mostrar éstas diferencias estadísticas. Para comprobar directamente si la insulina estimula la proliferación en estos cultivos, se calculó el porcentaje de IM de los cultivos NT-1 suplementados con $0.225 \mu \mathrm{M}$ de auxinas e insulina a diferentes concentraciones, el máximo IM obtenido que fue de un $6 \%$ se observó con $1.23 \mathrm{nM}$ de insulina, el cual se incrementó un $20 \%$ respecto al control (Figura 4C). Aun así, el 6\% de IM alcanzado por adición de insulina no se asemejó al obtenido en medios de cultivo suplementados con $0.9 \mathrm{nM}$ de auxinas y $1.23 \mathrm{nM}$ de insulina que alcanzó un 14\% (Figura 1A), lo que indica que la proliferación de los cultivos NT-1 es dependiente de la concentración de auxinas. El tamaño de las células de los cultivos suplementados con $1.23 \mathrm{nM}$ de insulina fue menor respecto al control, fenotipo que se asocia con células que se encuentran proliferando (Figuras 4D-G) (Nagata, 2004).

Debido a que la insulina estimuló la proliferación en los cultivos suplementados con $0.225 \mu \mathrm{M}$ de 2,4-D, se evaluó la expresión de los genes ciclina $B, C D K B, E 2 F$ que regulan directamente el ciclo celular y de los genes para la subunidad $\beta-G A R C A$ y $G S T$ que lo hacen indirectamente. En la Figura 5 se observa que 1.23 $\mathrm{nM}$ de insulina incrementó los niveles de los transcritos de ciclina $B, C D K B, E 2 F$ y GST y disminuyo la expresión de la subunidad $\beta$ - $G$, está última se sabe queregula negativamente la proliferación celular en Arabidopsis (Ullah et al., 2003). Es decir que la insulina estimula la proliferación en cultivos limitados de dicha actividad a través de la regulación transcripcional de algunos reguladores del ciclo celular.
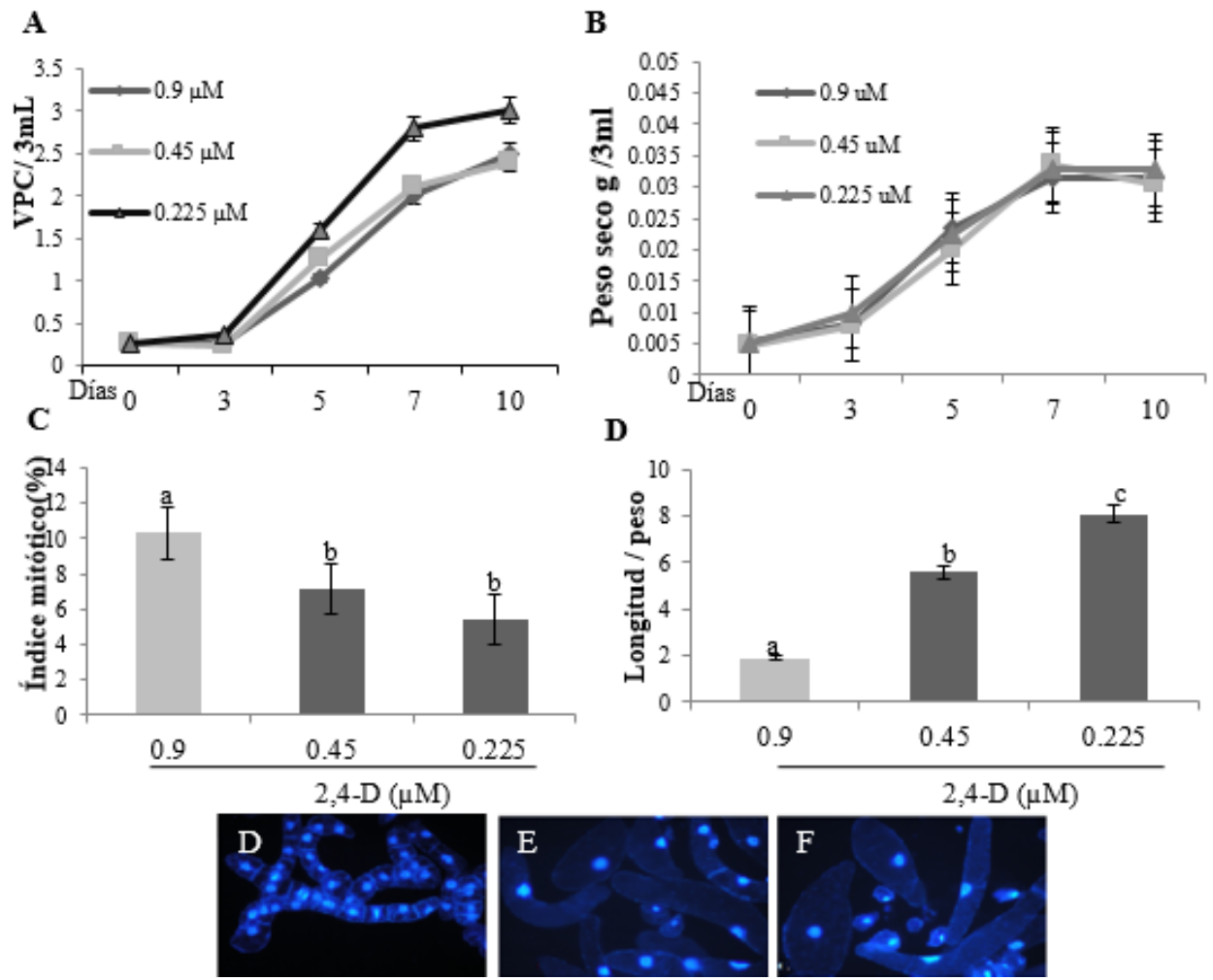

Figura 3. Efecto de diferentes concentraciones de 2,4-D sobre el crecimiento de los cultivos NT-1. Cinéticas de crecimiento de los cultivos suplementados con 0.9, 0.45 y $0.225 \mu \mathrm{M}$ de 2,4-D evaluados por A) VPC y B) Peso seco. C) Porcentaje de Índice Mitótico (IM) determinado en el día seis de fase log. D) Tamaño celular. Las células teñidas con DAPI se midieron para calcular la relación largo/ancho con el programa ImageJ. Fotografías de células NT-1 en fase $\log$ teñidas con DAPI. Tukey E) control, F) $0.45 \mu \mathrm{M}$ y G) $0.225 \mu \mathrm{M}$. P $\leq 0.05$; $\mathrm{n}=4$ STATISTICA ver. 8.0. 


\section{G. Fierros-Romero et al.}

A
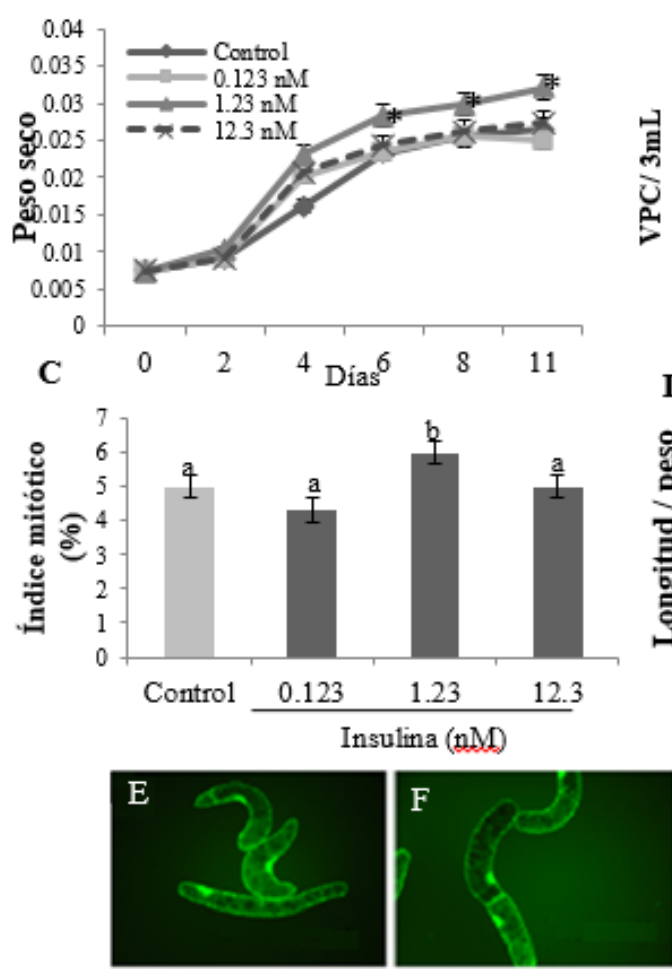

B
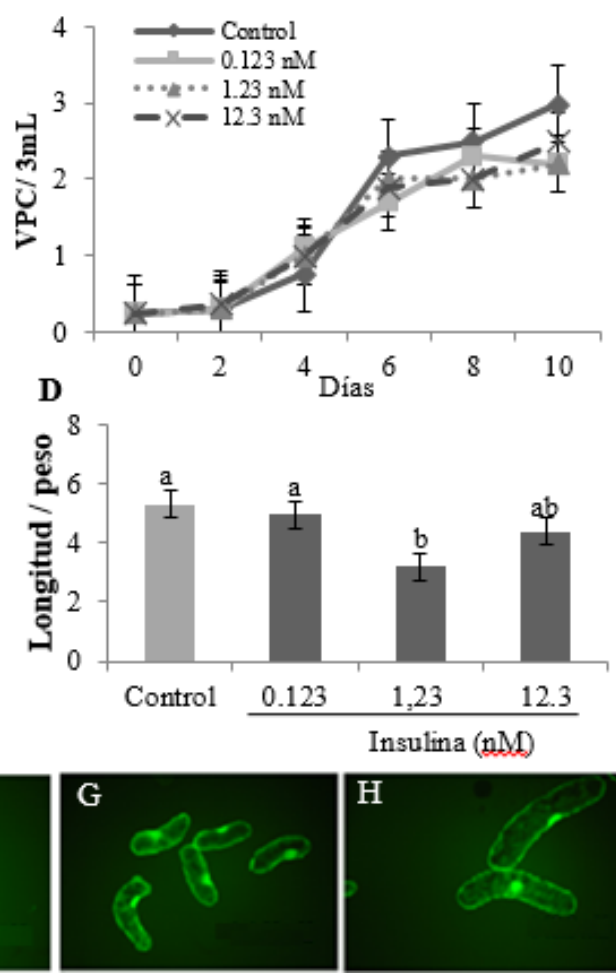

$\mathrm{H}$

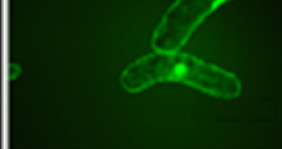

Figura 4. Efecto de la insulina sobre el crecimiento de los cultivos NT-1 con baja concentración de auxina. A) Cinéticas de crecimiento de los cultivos con $0.225 \mu \mathrm{M}$ de 2,4-D y suplementados con $0.123,1.23$ y $12.3 \mathrm{nM}$ de insulina evaluadas por A) peso seco y B) VPC. C) Porcentaje de Índice Mitótico (IM) determinado en el día seis de fase log. D) Tamaño celular. Las células teñidas con DAPI se midieron para calcular la relación largo/ancho con el programa ImageJ. Fotografías de células NT-1 en fase log teñidas con DFA. E) Control, F) Células suplementadas con $0.123 \mathrm{nM}$ de insulina, G) $1.23 \mathrm{nM} \mathrm{y} \mathrm{H)} 12.3 \mathrm{nM}$. Tukey. $\mathrm{P} \leq 0.05$; $\mathrm{n}=4$ STATISTICA ver. 8.0. 


\section{G. Fierros-Romero et al.}

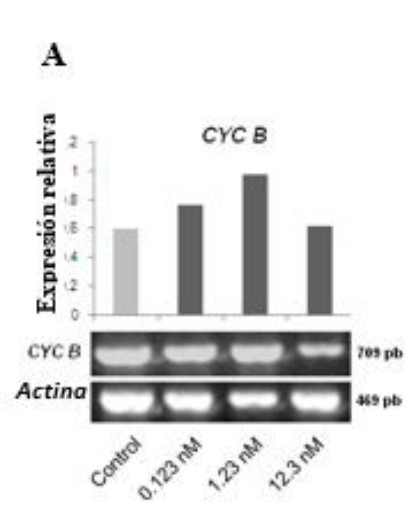

D
B

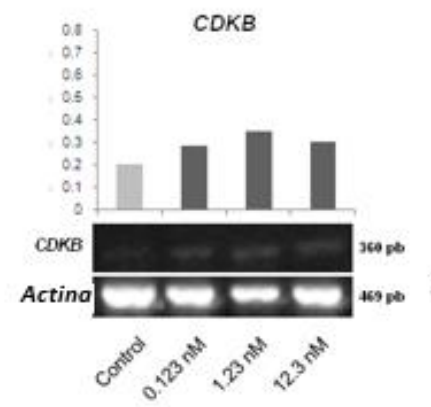

$\mathrm{C}$

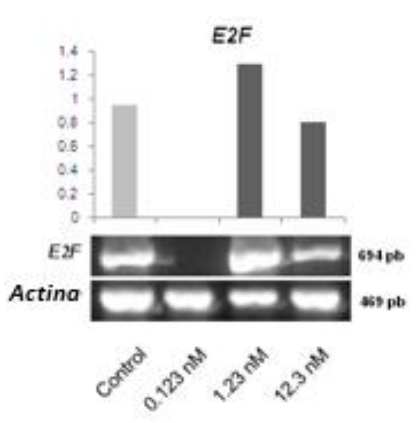

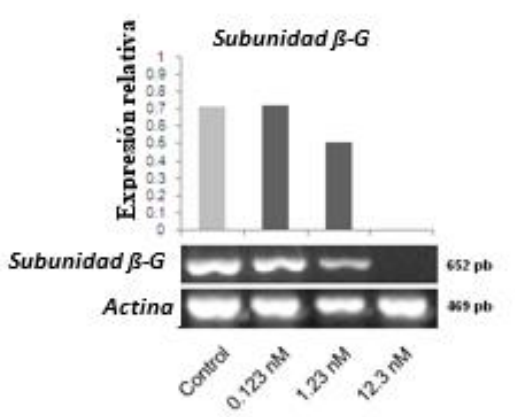

$\mathbf{E}$

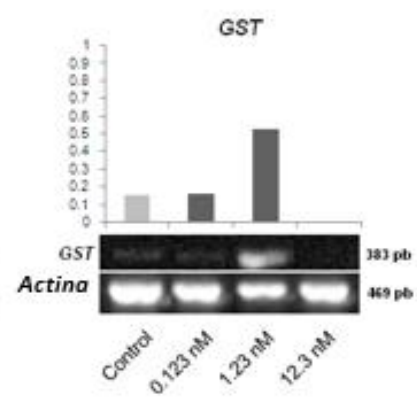

Figura 5. Efecto de la insulina sobre la expresión de genes reguladores del ciclo celular en los cultivos NT-1 con baja concentración de auxina. Los cultivos con $0.22510 \mu \mathrm{M}$ fueron suplementados con 0.123, 1.23 y $12.3 \mathrm{nM}$ de Se realizó un análisis de RT-PCR semi cuantitativo de los genes A) ciclina $B$, B) $C D K B$, C) E2F, D) subunidad $\beta$ - G ARC A, E) GST y actina como control de carga. Las fotografías mostradas representan el análisis de los productos de amplificación de cada gen en gel de agarosa $1 \%$.

3.4. La insulina estimula la proliferación celular en cultivos parcialmente sincronizados por privación de auxinas

Debido a que las auxinas determinan el efecto de la insulina sobre la proliferación, se decidió evaluar dicho efecto en un sistema parcialmente sincronizado por ausencia de auxinas. Se ha descrito en cultivos BY-2 que la privación de auxinas induce que la mayoría de las células se arresten en G1, dando lugar a una sincronización parcial y a células elongadas como consecuencia de la escasa división (Chen et al., 2001; Ishida et al., 1993). En los cultivos de tabaco privados de auxinas se han reportado células alargadas como consecuencia de una expansión isodiamétrica provocada por una mayor entrada de agua a las células (Nagata, 1992; Ulrich y Key, 1988; Winicur et al., 1998).

Se privó a los cultivos de 2,4-D durante cuatro días al cabo de los cuales, fueron suplementados con auxinas. En la Figura 6 se muestra que tanto el crecimiento evaluado por peso seco como el IM se incrementaron en presencia de insulina. Debido a la estimulación en este último parámetro, se evaluó la expresión de algunos genes reguladores del ciclo celular bajo estas condiciones. Se muestra en la Figura 7 que la insulina estimuló la expresión del gen $E 2 F$, lo que sugiere que en las células parcialmente sincronizadas que permanecen en la fase G1 del ciclo celular, la insulina induce la proliferación a través de la transcripción de dicho gen. 


\section{G. Fierros-Romero et al.}

A

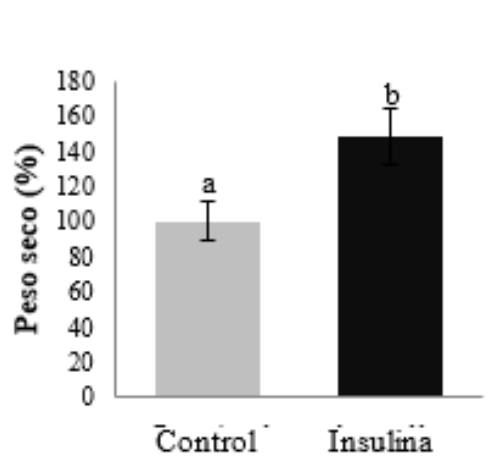

B

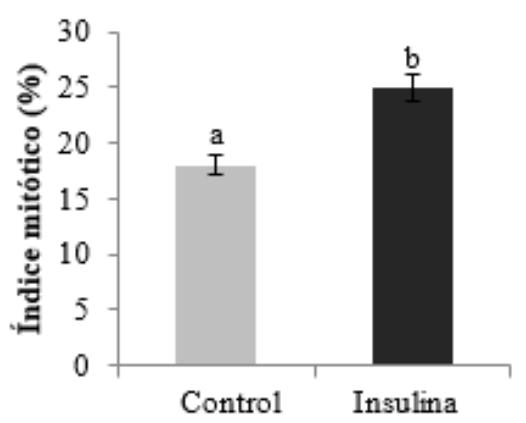

Figura 6. Efecto de la insulina sobre el crecimiento de los cultivos NT-1 parcialmente sincronizados. A) Se determinó el porcentaje de crecimiento de los cultivos NT-1 suplementados con $1.23 \mathrm{nM}$ de insulina al día seis de la fase log de la cinética de crecimiento por peso seco. B) Porcentaje de Índice Mitótico (IM) determinado en el día seis de fase log. Tukey $\mathrm{P} \leq 0.05 ; \mathrm{n}=4$ STATISTICA ver. 8.0.

A

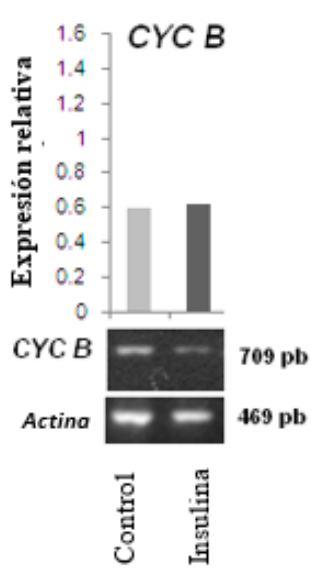

B

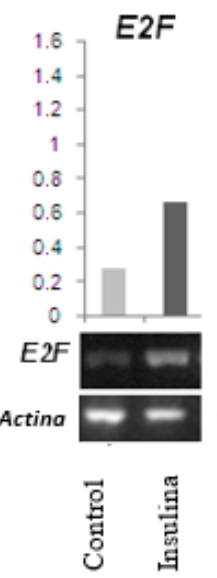

C

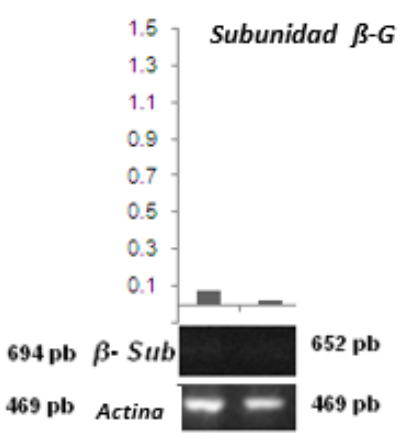

总㺃

Figura 7. Efecto de la insulina sobre la expresión de genes reguladores del ciclo celular en cultivos NT-1 parcialmente sincronizados. Los cultivos se suplementaron con $1.23 \mathrm{nM}$ de insulina Se realizó un análisis de RT-PCR semi cuantitativo de los genes A) ciclina $B, \mathrm{~B}) E 2 F$, C) subunidad $\beta$ - $G$ y actina como control de carga. Las fotografías mostradas representan el análisis de los productos de amplificación de cada gen en gel de agarosa $1 \%$.

\section{DISCUSIÓN}

Diferente evidencia sugiere que la insulina estimula el crecimiento en plantas como maíz y frijol (Sánchez de Jiménez et al., 1999; Santos, 2003) a una concentración de 1.23 nM. En maíz dicha concentración promovió la división y el crecimiento celular a través del reclutamiento a polisomas de transcritos como ciclina D y PCNA (Sotelo et al., 2010). En otros estudios sobre maíz, dicha concentración aceleró la germinación y la fosforilación de la proteína ribosomal S6 (Sánchez de Jiménez et al., 1999). En este estudio, los cultivos NT-1 bajo el efecto de $1.23 \mathrm{nM}$ de insulina fueron estimulados en la proliferación celular, a través de un incremento de biomasa e IM (Figuras suplementarias 1 y 2), estos datos sugieren que la insulina participa en los procesos de división de los cultivos NT-1, probablemente a través de la regulación del ciclo celular. Nuestros resultados indican que los cultivos NT-1 son capaces de responder a la insulina a la concentración de $1.23 \mathrm{nM}$ ya reportada para promover el crecimiento en otros sistemas vegetales de forma eficaz. Por otra parte, las auxinas comprenden una clase de reguladores del crecimiento vegetal importantes durante la morfogénesis celular dependiente de su concentración (Simon y Petrasek, 2011). Los

Nexo Revista Científica / Vol. 29, No. 02, pp. 44-58 / Diciembre 2016 


\section{G. Fierros-Romero et al.}

cultivos NT-1 responden directamente a la concentración de auxinas, ya que la auxina sintética 2,4-D estimula la división y a concentraciones limitantes induce la elongación (Nagata et al., 2004). Aquí, encontramos que los cultivos NT-1 bajo el efecto de la insulina requirieron de la presencia de 2,4-D durante la proliferación, debido al escaso crecimiento observado sin 2,4-D (Figura 2). Se ha reportado que ambas hormonas, la insulina y las auxinas estimularon el crecimiento en maíz induciendo la fosforilación de la proteína ribosomal S6 por rutas transduccionales indepedientes (Buentello Volante et al., 2010). Como sabemos, las auxinas son determinantes para la proliferación de los cultivos de tabaco BY-2 y NT-1 (Nagata, 2004), principalmente durante la progresión del ciclo en G1/S (Ito et al., 1998) y presentando una expansión por la escasa actividad mitótica (Chen et al., 2001; Nagata, 1992). En otras líneas celulares de tabaco como la VBI se ha demostrado que las auxinas como el ácido naftalen acético (ANA) y el 2,4-D, regulaban la proliferación y la elongación diferencialmente. El ANA estimuló la elongación y el 2,4-D promovía la división, proponiendo los autores dos rutas de respuesta a auxinas diferentes dependientes de la concentración para el control de la división y la elongación en estos sistemas. En relación a lo anterior se propuso que ANA puede actuar a través del receptor ABP1, mientras que el 2,4D puede utilizar uno acoplado a proteínas $\mathrm{G}$ heterotriméricas.

Cuando las células vegetales crecen en condiciones de baja concentración de auxinas se presenta un incremento de cisternas en el trans-Golgi (Winicur et al., 1998), acumulación de almidón (Miyazawa et al., 1999), cambios en la presión osmótica y en la expansión celular (Chen et al., 2001). En los cultivos con escasa actividad mitótica como consecuencia de una baja concentración de auxinas, la insulina incrementó la proliferación (Figura 4) y la expresión de genes reguladores directos del ciclo celular como ciclina $B$, $C D K B, E 2 F$ e indirectos como la subunidad $B-G$ y $G S T$ (Figura 5). La expresión de ciclina $B$ y CDKB se presenta en la transición G2/M (Ito et al., 2001; Sorrell et al., 2001), E2F se expresa en G1/S (Magyar et al., 2005) y GST es un gen de respuesta temprana a auxina presente en la fase $\mathrm{S}$ del ciclo celular (Takahashi y Nagata, 1992). Mientras que las subunidad $\beta-G$ tiende a disminuir su expresión cuando hay altas cantidades de auxinas, siendo dicho transcrito un regulador negativo de la proliferación(Ullah et al., 2003). Los resultados anteriores sugieren que los cultivos con baja concentración de auxinas y escasa actividad mitótica, bajo el efecto de la insulina restablecen la proliferación estimulando la progresión del ciclo celular a través de la regulación transcripcional de genes que regulan la división.

En cultivos de maíz sin restricción de fitorreguladores, también se ha descrito que la insulina estimula la proliferación celular a través ciclina D y PCNA (Sotelo et al., 2010). Por otra parte, se ha reportado que en cultivos BY-2 la ausencia de auxina conduce a una sincronización parcial con una proliferación acelerada después de la re-adición de auxinas al medio (Ishida et al., 1993). Aquí observamos que la insulina estimula el crecimiento de los cultivos previamente privados de auxina, a través de la expresión de $E 2 F$ en la transición GI/S (Figura 7). En relación a lo anterior, se ha descrito que la sobre expresión del factor E2F en cultivos BY-2 privados de auxina promueve la proliferación celular, presentando células pequeñas correspondientes a un estado activo de división (Magyar et al., 2005). En los cultivos BY-2 carentes totalmente de auxinas, las células se estancan en G1 del ciclo celular y la muerte celular se presenta después del cuarto día (Mlejnek y Prochazka, 2002). Este tipo de cultivos parcialmente sincronizados presentan diferencias importantes respecto a los cultivos previamente analizados como: el acortamiento de la fase lag (datos no presentados) y un mayor índice mitótico (Figura 6B). Se ha reportado que en cultivos BY-2 bajo estas condiciones, se presenta una proliferación acelerada después de la re-adición de auxinas al medio (Nagata, 2004). En conjunto los datos sugieren que las respuestas a la insulina de los cultivos NT-1 a durante la proliferación celular son dependientes de la vía auxínica coriente arriba de la transcripción genética y que existe respuestas diferenciales de los cultivos NT-1 dependientes de auxinas.

\section{CONCLUSIÓN}

El crecimiento y desarrollo vegetal involucra la integración de diversas señales ambientales y endógenas que determinan el desarrollo de la planta. En cultivos de tabaco NT-1 las auxinas dependientes de su

Nexo Revista Científica / Vol. 29, No. 02, pp. 44-58 / Diciembre 2016 


\section{G. Fierros-Romero et al.}

concentración son determinantes para los procesos de proliferación y elongación. Sin embargo, hormonas animales como la insulina pueden desencadenar respuestas durante el crecimiento de plantas, remarcando la importancia de mecanismos ampliamente conservados entre diferentes reinos. En los cultivos NT-1 en suspensión, la insulina fue capaz de estimular la proliferación celular de forma dependiente de la concentración de auxinas y la regulación de genes durante este proceso. Siendo además este sistema, un excelente modelo para estudiar la función de la insulina en plantas a través de la vía auxínica.

\section{FIGURAS SUPLEMENTARIAS}

A

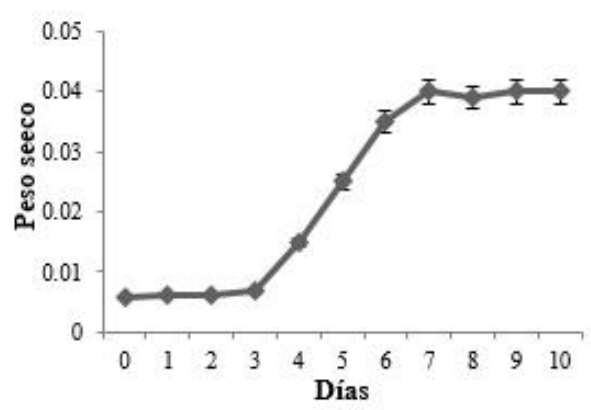

B

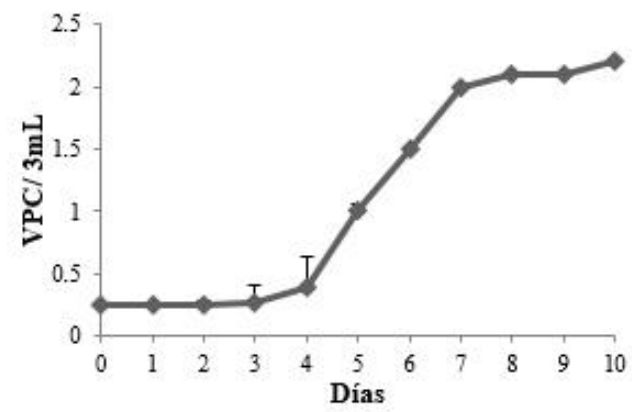

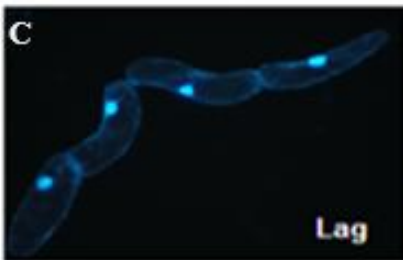
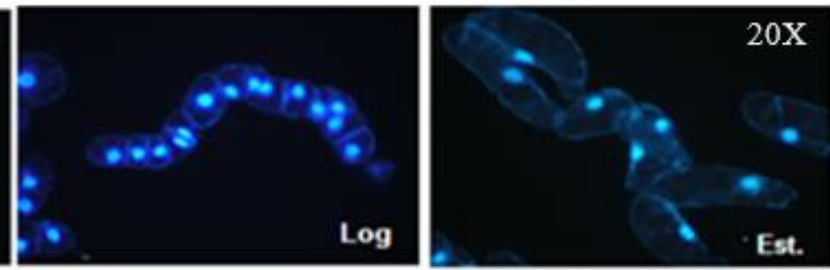

Figura suplementaria 1. Cinética de crecimiento de los cultivos NT-1. Las cinéticas de crecimiento se determinaron analizando el crecimiento diariamente a través de alícuotas de $3 \mathrm{~mL}$ para determinar el A) peso seco y B) Volumen de Paquete Celular (VPC). C) Imágenes representativas de las células NT-1 teñidas con DAPI en las diferentes fases de crecimiento, donde se presentan células alargadas en las fases lag y estacionaria, y células pequeñas en la fase $\log$. P $\leq$ $0.05 ; \mathrm{n}=4$ STATISTICA ver. 8.0 .

A

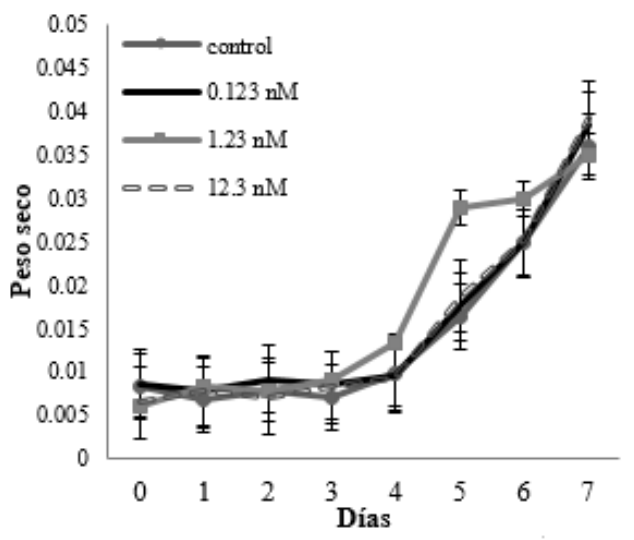

B

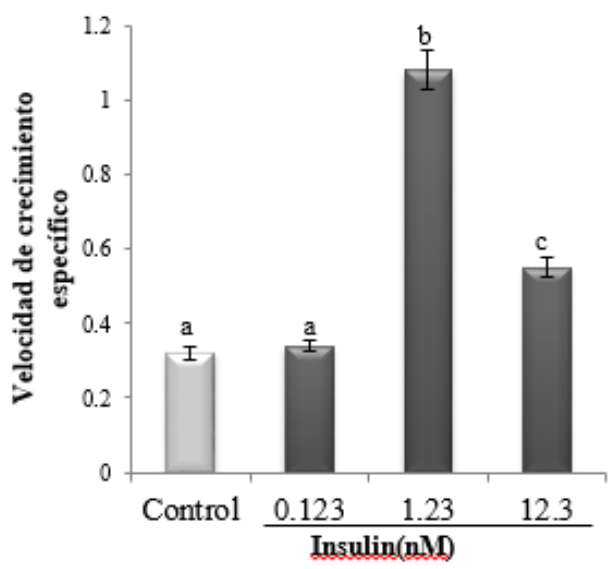

Figura suplementaria2. Efecto de diferentes concentraciones de insulina sobre el crecimiento de los cultivos NT-1. A) Cinéticas de crecimiento de los cultivos suplementados con insulina. Se realizaron cinéticas de crecimiento analizando diariamente el crecimiento de cultivos suplementados con $0.123,1.23,12.3$ y $61.5 \mathrm{nM}$ de insulina por peso seco. Tukey $\mathrm{P} \leq 0.05 ; \mathrm{n}=4$ STATISTICA ver. 8.0. B) Velocidad específica de crecimiento de los cultivos NT-1 a diferentes 


\section{G. Fierros-Romero et al.}

concentraciones de insulina. La velocidad específica de crecimiento se calculó de las cinéticas de crecimiento evaluadas por peso seco de cultivos suplementados con $0.123,1.23$ y $12,3 \mathrm{nM}$ de insulina, mediante la ecuación: $\mu=$ $\ln \mathrm{X}-\ln \mathrm{X}_{0} / t$,donde $\mu$ es la velocidad específica de crecimiento, $\mathrm{X}_{0}$ es la biomasa inicial, $\mathrm{X}$ es la final y t el tiempo comprendido entre el inicio y el final de la fase $\log$. Tukey $\mathrm{P} \leq 0.05 ; \mathrm{n}=4$ STATISTICA ver. 8.0

\section{REFERENCIAS}

Best, C. S., M.A. (1923). Possible sources of insulin. Metabol Res 3, 177-179.

Buentello Volante, B., Díaz de León-Sánchez, F., Rivera-Cabrera, F., Aguilar Caballero, R., Ponce-Valadez, M., Sánchez de Jiménez, E., y Pérez-Flores, L. (2010). Apparent cross-talk of two signaling pathways that regulate Zea mays coleoptile growth. Phyton (Buenos Aires) 79, 101-108.

Collip, J. (1923). Glucokinin. A new hormone present in plant tissue. J Biol Chem 56, 513-543.

Chen, J. G., Ullah, H., Young, J. C., Sussman, M. R., y Jones, A. M. (2001). ABP1 is required for organized cell elongation and division in Arabidopsis embryogenesis. Genes \& Development 15, 902-911.

Garcia Flores, C., Aguilar, R., Reyes de la Cruz, H., Albores, M., y Sanchez de Jimenez, E. (2001). A maize insulin-like growth factor signals to a transduction pathway that regulates protein synthesis in maize. The Biochemical journal 358, 95-100.

Goodman, D. B., y Davis, W. L. (1993). Insulin accelerates the post germinative development of several fat-storing seeds. Biochemical and biophysical research communications 190, 440-446.

Gutierrez, C. (2009). The Arabidopsis cell division cycle. Arabidopsis Book 7, 20.

Ishida, S., Takahashi, Y., y Nagata, T. (1993). Isolation of cDNA of an auxin-regulated gene encoding a G protein beta subunit-like protein from tobacco BY-2 cells. Proc Natl Acad Sci U S A 90, 11152-11156.

Ito, M., Araki, S., Matsunaga, S., Itoh, T., Nishihama, R., Machida, Y., Doonan, J. H., y Watanabe, A. (2001). G2/M-phase-specific transcription during the plant cell cycle is mediated by c-Myb-like transcription factors. Plant Cell 13, 1891-1905.

Ito, M., Iwase, M., Kodama, H., Lavisse, P., Komamine, A., Nishihama, R., Machida, y., and Watanabe, A. (1998). A novel cis-acting element in promoters of plant B-type cyclin genes activates $M$ phase-specific transcription. Plant Cell 10,331-341.

Jimenez-Lopez, S., Mancera-Martinez, E., Donayre-Torres, A., Rangel, C., Uribe, L., March, S., JimenezSanchez, G., y Sanchez de Jimenez, E. (2011). Expression profile of maize (Zea mays L.) embryonic axes during germination: translational regulation of ribosomal protein mRNAs. Plant \& cell physiology 52, 17191733.

Magyar, Z., De Veylder, L., Atanassova, A., Bakó, L., Inzé, D., y Bögre, L. (2005). The Role of the Arabidopsis E2FB Transcription Factor in Regulating Auxin-Dependent Cell Division. Plant Cell 17, 25272541.

Matsuoka, K., Demura, T., Galis, I., Horiguchi, T., Sasaki, M., Tashiro, G., y Fukuda, H. (2004). A comprehensive gene expression analysis toward the understanding of growth and differentiation of tobacco BY-2 cells. Plant \& cell physiology 45, 1280-1289. 


\section{G. Fierros-Romero et al.}

Miyazawa, Y., Sakai, A., Miyagishima, S., Takano, H., Kawano, S., y Kuroiwa, T. (1999). Auxin and Cytokinin Have Opposite Effects on Amyloplast Development and the Expression of Starch Synthesis Genes in Cultured Bright Yellow-2 Tobacco Cells. Plant Physiology 121, 461-470.

Mlejnek, P., y Prochazka, S. (2002). Activation of caspase-like proteases and induction of apoptosis by isopentenyladenosine in tobacco BY-2 cells. Planta 215, 158-166.

Mockaitis, K., y Estelle, M. (2008). Auxin receptors and plant development: a new signaling paradigm. Annu Rev Cell Dev Biol 24, 55-80.

Mravec, J., Kubes, M., Bielach, A., Gaykova, V., Petrasek, J., Skupa, P., Chand, S., Benkova, E., Zazimalova, E., y Friml, J. (2008). Interaction of PIN and PGP transport mechanisms in auxin distributiondependent development. Development (Cambridge, England) 135, 3345-3354.

Nagata, T. (2004). When I encountered tobacco BY-2 cells! . In Biotechnology in Agriculture and Forestry. T.H. Nagata, S; Inzé, D, ed. (New York: Springer), pp. 1-5.

Nagata, T., Sakamoto, K., y Shimizu, T. (2004). Tobacco by-2 cells: The present and beyond. In Vitro Cellular \& Developmental Biology - Plant 40, 163-166.

Nagata, T. N., y; Hasezawa, S (1992). Tobacco BY-2 cell line as the "HeLa" cell in the cell bioloby of higher plants. Int Rev Cytol 132, $1-30$.

Perrot-Rechenmann, C. (2010). Cellular responses to auxin: division versus expansion. Cold Spring Harb Perspect Biol 2, 7.

Raghavan, V. (2004). Role of 2,4-dichlorophenoxyacetic acid (2,4-D) in somatic embryogenesis on cultured zygotic embryos of Arabidopsis: cell expansion, cell cycling, and morphogenesis during continuous exposure of embryos to 2,4-D. American journal of botany 91, 1743-1756.

Rodriguez-Lopez, C. D., Rodriguez-Romero, A., Aguilar, R., y de Jimenez, E. S. (2011). Biochemical characterization of a new maize (Zea mays L.) peptide growth factor. Protein and peptide letters 18, 84-91.

Saltiel, A. R., y Kahn, C. R. (2001). Insulin signalling and the regulation of glucose and lipid metabolism. Nature 414, 799-806.

Sánchez de Jiménez, E., Beltrán-Peña, E., y Ortíz-López, A. (1999). Insulin-stimulated ribosomal protein synthesis in maize embryonic axes during germination. Physiologia Plantarum 105, 148-154.

Santos, V. (2003) Presenca de insulin em phaseolus vulgaris L. cv. Carioca, Monograph, Universidade Estadual Norte Fluminense Campos dos Goytacazes. R.J., Simon, S., y Petrasek, J. (2011). Why plants need more than one type of auxin. Plant science : an international journal of experimental plant biology 180, 454460.

Sorrell, D. A., Menges, M., Healy, J. M., Deveaux, Y., Amano, C., Su, y., Nakagami, H., Shinmyo, A., Doonan, J. H., Sekine, M., y Murray, J. A. (2001). Cell cycle regulation of cyclin-dependent kinases in tobacco cultivar Bright Yellow-2 cells. Plant Physiol 126, 1214-1223.

Sotelo, R., Garrocho-Villegas, V., Aguilar, R., Calderón, M., y de Jiménez, E. (2010). Coordination of cell growth and cell division in maize (Z\&lt;i\&gt;ea mays\&lt;/i\&gt; L.) relevance of the conserved TOR signal transduction pathway. In Vitro Cellular \& Developmental Biology - Plant 46, 578-586.

Nexo Revista Científica / Vol. 29, No. 02, pp. 44-58 / Diciembre 2016 


\section{G. Fierros-Romero et al.}

Takahashi, Y., y Nagata, T. (1992). parB: an auxin-regulated gene encoding glutathione S-transferase. Proc Natl Acad Sci U S A 89, 56-59.

Ulrich, T. H., y Key, J. L. (1988). Comparative Analysis of Polyadenylated RNA Complexity in Soybean Hypocotyl Tissue and Cultured Suspension Cells. Plant Physiology 86, 482-490.

Ullah, H., Chen, J. G., Temple, B., Boyes, D. C., Alonso, J. M., Davis, K. R., Ecker, J. R., y Jones, A. M. (2003). The beta-subunit of the Arabidopsis G protein negatively regulates auxin-induced cell division and affects multiple developmental processes. Plant Cell 15, 393-409.

Winicur, Z. M., Zhang, G. F., y Staehelin, L. A. (1998). Auxin deprivation induces synchronous Golgi differentiation in suspension-cultured tobacco BY-2 cells. Plant Physiol 117, 501-513.

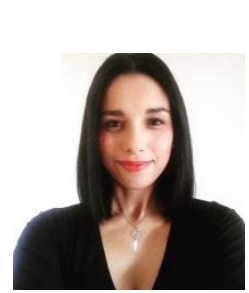

\section{SEMBLANZA DE LOS AUTORES}

Grisel Fierros Romero. Actualmente realiza un postdoctorado en Florida Agricultural and Mechanical University en Florida, USA. Su investigación se desarrolla en el área de microbiología ambiental, estudiando interacciones de bacterias predadoras de origen marino con bacterias del género Vibrio y protistas. Obtuvo el grado de doctorado en Tecnología Avanzada en el Instituto Politécnico Nacional y el grado de maestría en Biología Experimental en la Universidad Michoacana de San Nicolás de Hidalgo. Es Química Farmacobióloga de formación y obtuvo el grado en la UMSNH. Realizo una estancia de investigación en la Universidad Friedrich Schiller en Jena, Alemania y ha participado en congresos nacionales e internacionales. Ha participado en diferentes líneas de investigación que incluyen, biotecnología vegetal, farmacología, microbiología ambiental, bioinformática, biología molecular y resistencia a metales pesados.

Maria Elena Mellado-Rojas. Trabaja con biogénesis del ribosoma en la regulación del crecimiento y división en células de tabaco (plantas y cultivos en suspensión de Arabidopsis).

Elda Maria Beltrán-Peña Investigadora en el Instituto de Investigaciones Quimico-Biologicas en Morelia, Michoacan. Desarrolla investigación en biogénesis del ribosoma en la regulación del crecimiento y división en células de tabaco (plantas y cultivos en suspensión de Arabidopsis). 